\title{
TRMM Precipitation Radar and Microwave Imager Observations of Convective and Stratiform Rain over Land and their Theoretical Implications
}

\author{
C. PRABHAKARA \\ NASA/Goddard Space Flight Center, Greenbelt, MD, USA \\ R. IACOVAZZI, Jr. \\ Science Systems and Applications, Inc., Lanham, MD, USA \\ and \\ J.-M. YOO \\ EWHA Womans University, Seoul, South Korea
}

(Manuscript received 21 November 2001, in revised form 25 June 2002)

\begin{abstract}
Observations of brightness temperature, $\mathrm{Tb}$, made over land regions by the Tropical Rainfall Measuring Mission (TRMM) Microwave Imager (TMI) radiometer are analyzed with the help of nearly simultaneous measurements of the vertical profiles of reflectivity factor, $Z$, made by the Precipitation Radar (PR) onboard the TRMM satellite. Furthermore, this analysis is done separately over convective and stratiform rain regions. This examination reveals a clear relationship between TMI and PR data. Possible explanation for this relationship is explored with the help of radiative transfer calculations. With this approach, we demonstrate that the $85 \mathrm{GHz}$ observations of TMI can be simulated crudely from the observations of $Z$. However, the 37 and $19 \mathrm{GHz}$ observations are not as well simulated, possibly because of horizontal non-uniformity in the hydrometeor distribution in the broad footprints of these channels and contamination introduced by land-surface emissivity. On the other hand, from TMI and PR observations, we find that the brightness temperature difference (T19-T37) minimizes these sources of error. Our simulations of (T19-T37) over convective rain regions are in reasonable agreement with this finding. This investigation indicates that the TMI $85 \mathrm{GHz}$ channel yields the best information about rain over tropical land, because it has minimal surface contamination, strong extinction, and a fine footprint. The brightness temperature difference (T19-T37) can supplement the information given by the $85 \mathrm{GHz}$ channel.
\end{abstract}

\section{Introduction}

Observational studies of convective and stratiform rain reveal that these two types

Corresponding author: C. Prabhakara, NASA/ Goddard Space Flight Center, Code 913, Greenbelt, Maryland 20771, USA.

E-mail: cuddapah@climate.gsfc.nasa.gov

(C) 2002, Meteorological Society of Japan of rain are associated with distinctly different types of hydrometeors in a vertical column of the atmosphere (e.g., Houze 1993, 1997). This is mainly because of differences in the dynamics and the physical processes associated with these two rain types. This observational background helps us to investigate the satellite measurements from the Tropical Rainfall Measuring Mission (TRMM) Microwave Imager 
(TMI) radiometer and Precipitation Radar (PR) over convective and stratiform rain regions.

Onboard the TRMM satellite, the TMI and PR produce nearly simultaneous observations over ocean and land under different weather conditions. The brightness temperatures, Tbs, measured by TMI represent upwelling microwave radiation that has undergone interaction with atmospheric oxygen, water vapor, cloud liquid water, and liquid and frozen hydrometeors in a vertical column of the atmosphere. On the other hand, the measurements of reflectivity factor $Z$ (from hereafter referred to as reflectivity) made by $\mathrm{PR}$ near $13.8 \mathrm{GHz}$ depend upon the backscatter of microwave radiation, and have a vertical resolution of $250 \mathrm{~m}$. Reflectivity observations near the surface can yield an estimate of the rain rate at that level when a suitable particle size distribution (PSD) is assumed. Based on the above discussion, one expects to find a relationship between the vertical profiles of $Z$ observed from PR and the Tb measurements of TMI. The objective of this study is to reveal such a relationship with the help of TMI and PR data, and then explain that relationship theoretically.

Before the launch of the TRMM satellite, a feasibility study made by Haddad et al. (1997) showed that the vertical profile of rain could be deduced from a combination of the PR and TMI data. In another study, in order to understand the link between microwave radiometer and radar observations, Bennartz and Petty (2001) performed an analysis combining the brightness temperatures observed by Special Sensor Microwave Imager radiometer (flown on Defense Meteorological Satellite Program satellites) and measurements of reflectivity made by a surface radar over the Baltic Sea near $57^{\circ} \mathrm{N}$. Their study reveals the effect of variable size distribution and density of precipitating ice particles on microwave Tbs.

Viltard et al. (2000) examined the importance of the PSD in linking the PR data with the TMI measured Tbs over oceans utilizing radiative transfer simulations. The vertical profiles of the water content in the atmosphere, needed in those simulations, were selected from a table of profiles generated with the help of a cloud resolving model. However, the selected profiles of the water content were not in complete agreement with the $\mathrm{PR}$ reflectivity profiles. Adopting that approach, Viltard et al. were able to show crudely the significance of PSD over ocean.

In this study, we probe into the connection between the TRMM PR and TMI observations over land following a different approach that emphasizes the measurements of these two instruments. First, we have compared the vertical profiles of $Z$ given by the PR with the Tbs in the 19, 37, and $85 \mathrm{GHz}$-i.e., T19, T37, and T85-measured by the TMI. This is done separately for convective and stratiform rain areas, which are discriminated by the PR as indicated in the 2A25 TRMM product (see Iguchi et al. 2000). This analysis of observations is useful to reveal the inherent relationship between the PR and TMI data. Then, hydrometeor profiles that are consistent with the $Z$ profiles are formed with guidance from several past studies of the microphysical structure in convective and stratiform rain regions. Utilizing these hydrometeor profiles, radiative transfer calculations are made to simulate the Tbs observed by TMI. From this investigation, we can infer the relative importance of different channels of the TMI radiometer for the purpose of rain retrieval over land.

\section{Correspondence between TMI and PR observations of convective and stratiform rain over land}

The near-surface PR observations have a horizontal resolution of about $4.3 \mathrm{~km} \times 4.3 \mathrm{~km}$. These PR data are arranged uniformly at a spacing of about $4.3 \mathrm{~km}$ both along and across scan lines. On the other hand, the footprints of the TMI $85 \mathrm{GHz}$ vertical and horizontal polarization channels are approximately $5.0 \mathrm{~km} \times$ $7.0 \mathrm{~km}$, and are separated by about $5.0 \mathrm{~km}$ along scan lines and about $14 \mathrm{~km}$ across scan lines. This scan pattern degrades the effective resolution of the TMI $85 \mathrm{GHz}$ channel compared to that of the PR. The TMI radiometer contains additional channels in vertical and horizontal polarization near 10,19 , and $37 \mathrm{GHz}$ that have a resolution of about 40,20 , and $10 \mathrm{~km}$, respectively. Furthermore, TMI has a $21 \mathrm{GHz}$ channel in vertical polarization that has a resolution similar to that of $19 \mathrm{GHz}$. In this study, we are interested in scrutinizing the information contained in the vertical profile of 

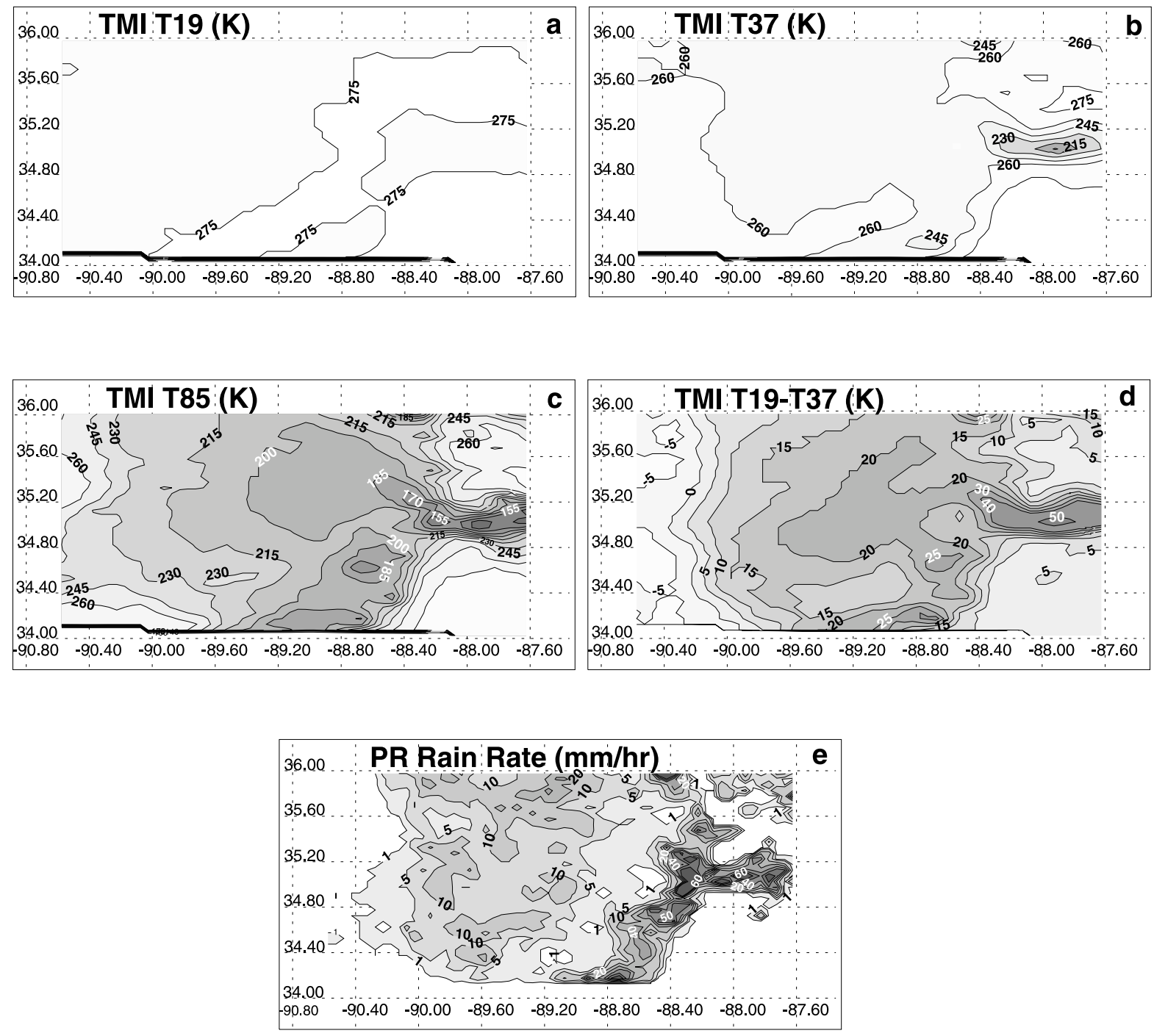

Fig. 1. Maps of a) T19, b) T37, c) T85, d) (T19-T37), and e) PR near-surface rain rate for a mesoscale convective system observed by the TRMM satellite on 5 June 1998 over the Southeast United States. Note that the brightness temperatures T19, T37, and T85 represent the vertical polarization measurements.

$Z$ measured by the PR and the Tbs measured by TMI in the 19,37 , and $85 \mathrm{GHz}$ channels. The TMI 10 and $21 \mathrm{GHz}$ channels are not included in this investigation. This is because the $10 \mathrm{GHz}$ channel has a relatively large footprint, and the information given by the $21 \mathrm{GHz}$ channel is very similar to that of $19 \mathrm{GHz}$. Also, we consider only the vertical polarization measurements made by TMI, because they tend to have less surface contamination compared to horizontal polarization measurements.

In Figs. 1a-e, we show maps of T19, T37,
T85, and (T19-T37), as well as a map of PR near-surface rain rate, for a mesoscale convective system (MCS) that occurred over the Southeast United States on 5 June 1998. From these figures, we note that the spatial distribution of $\mathrm{PR}$ rain rate compares well with that of T85, but poorly with that of T19 or T37. This is mainly because of the strong extinction due to hydrometeors in the $85 \mathrm{GHz}$ channel compared to that in the long wavelength channels near 37 and $19 \mathrm{GHz}$. The surface emission present in these long wavelength channels can degrade 
the rain information. However, a map of the difference, (T19-T37), which reduces this surface contamination, produces a good comparison with the $P R$ rain rate patterns. Such information given by the microwave radiometer and the radar is noticed over widely different regions of the tropics.

We show in Fig. 2a a vertical cross section of the radar reflectivity taken from TRMM PR along a sub-satellite track. This cross section, which starts at $\left(35.1^{\circ} \mathrm{N}, 90.6^{\circ} \mathrm{W}\right)$ and ends at $\left(35.0^{\circ} \mathrm{N}, 87.6^{\circ} \mathrm{W}\right)$, corresponds to the same MCS depicted in Figs. 1a-e. The PR reflectivity below the $1 \mathrm{~km}$ level is not shown in this figure to avoid problems related to signal clutter arising from surface topography. We note from Fig. 2a that the leading edge of the storm, with its tall convective towers, is near $88^{\circ} \mathrm{W}$. High reflectivity cores $(>30 \mathrm{dBZ})$ that extend well above the freezing level $(\sim 5.0 \mathrm{~km})$ characterize these convective towers. To the west of these convective towers, from $88.6^{\circ} \mathrm{W}$ to $90.3^{\circ} \mathrm{W}$, and above the freezing level, we notice an extensive anvil cloud with an almost horizontally uniform pattern of reflectivity. Below the freezing level, underneath the anvil cloud, there is a bright band that is about $0.5 \mathrm{~km}$ in thickness. Strong reflectivity in the bright band is attributed to large, low-density melting snow flakes, while the strong reflectivity above the freezing level in the cores of the convective towers is attributed to small, water-coated dense graupel (Bauer et al. 2000). Near the surface, there is intense convective rain in the convective towers, while there is relatively light stratiform rain underneath the anvil (see Fig. 2c). Notice there is a small region referred to as the Bounded Weak Echo Region (BWER) between $88.4^{\circ} \mathrm{W}$ and $88.5^{\circ} \mathrm{W}$ in the layer from $2 \mathrm{~km}$ to $6 \mathrm{~km}$, which is at the origin of the anvil cloud. Such a BWER has been described in earlier studies with the help of observations made by ground-based radars (see Houze 1993). This description of the reflectivity pattern and rain rate associated with a MCS is helpful in relating the TRMM PR observations with those of TMI.

In Fig. 2b, the brightness temperatures measured by TMI in the 85,37 and $19 \mathrm{GHz}$ channels are shown along the sub-satellite track to correspond to the cross section of PR reflectivity shown in Fig. 2a. In the response of the $85 \mathrm{GHz}$ channel, we find there is a strong scattering depression due to relatively dense ice particles (Wu and Weinman 1984) where the convective towers are present, but some of the individual towers are not well resolved. The anvil in the stratiform region produces weaker scattering depression. Over the entire cross-section domain, the response of the $37 \mathrm{GHz}$ channel is similar to that of the $85 \mathrm{GHz}$ channel, except that the scattering depression in $37 \mathrm{GHz}$ is not as strong. The response in the $19 \mathrm{GHz}$ channel is the weakest of the three channels. The interrelationship between TMI measured T85, T37, and T19, and the PR measured $Z$ and $R$, is only crudely reflected in Figs. 2a-c, because of differences in the resolution of the TMI and PR data.

To compare the TMI and PR data sets, we have averaged them to the TMI $19 \mathrm{GHz}$ field of view ( $f o v$ ), which has a size that is comparable to that of Cbs. As indicated earlier, the TMI scan data are not uniformly spaced. For this reason, to reduce the effect of non-uniformity in TMI data, we have applied a simple spatial averaging procedure.

The TMI has a conical scanning geometry, while the PR has a cross-track scanning geometry. These differences in scanning geometry can introduce spatial mismatch between PR and TMI data (Hong et al. 2000). The PR data near the surface is generally used to deduce rain information. On the contrary, the $85 \mathrm{GHz}$ temperature responds to the hydrometeors in the whole column of the atmosphere, with special emphasis on ice hydrometeors above the freezing level (Wu and Weinman 1984). Thus, differential advection of hydrometeors between the upper and lower levels can create spatial mismatch between the $85 \mathrm{GHz}$ data of TMI and PR near-surface rain observations. Also, the hydrometeors originating at higher altitudes take several minutes to reach the surface. For these reasons, averaging the data of each instrument to the $19 \mathrm{GHz}$ fov will reduce the problems involved in comparing the information content of these two datasets. Similar arguments also apply to the $37 \mathrm{GHz}$ data. However, compared to the $85 \mathrm{GHz}$, the $37 \mathrm{GHz}$ channel responds to lower layers of the atmosphere, while the $19 \mathrm{GHz}$ channel is sensitive to layers even further below. This is due to reduced ice scattering in these longer wavelength 

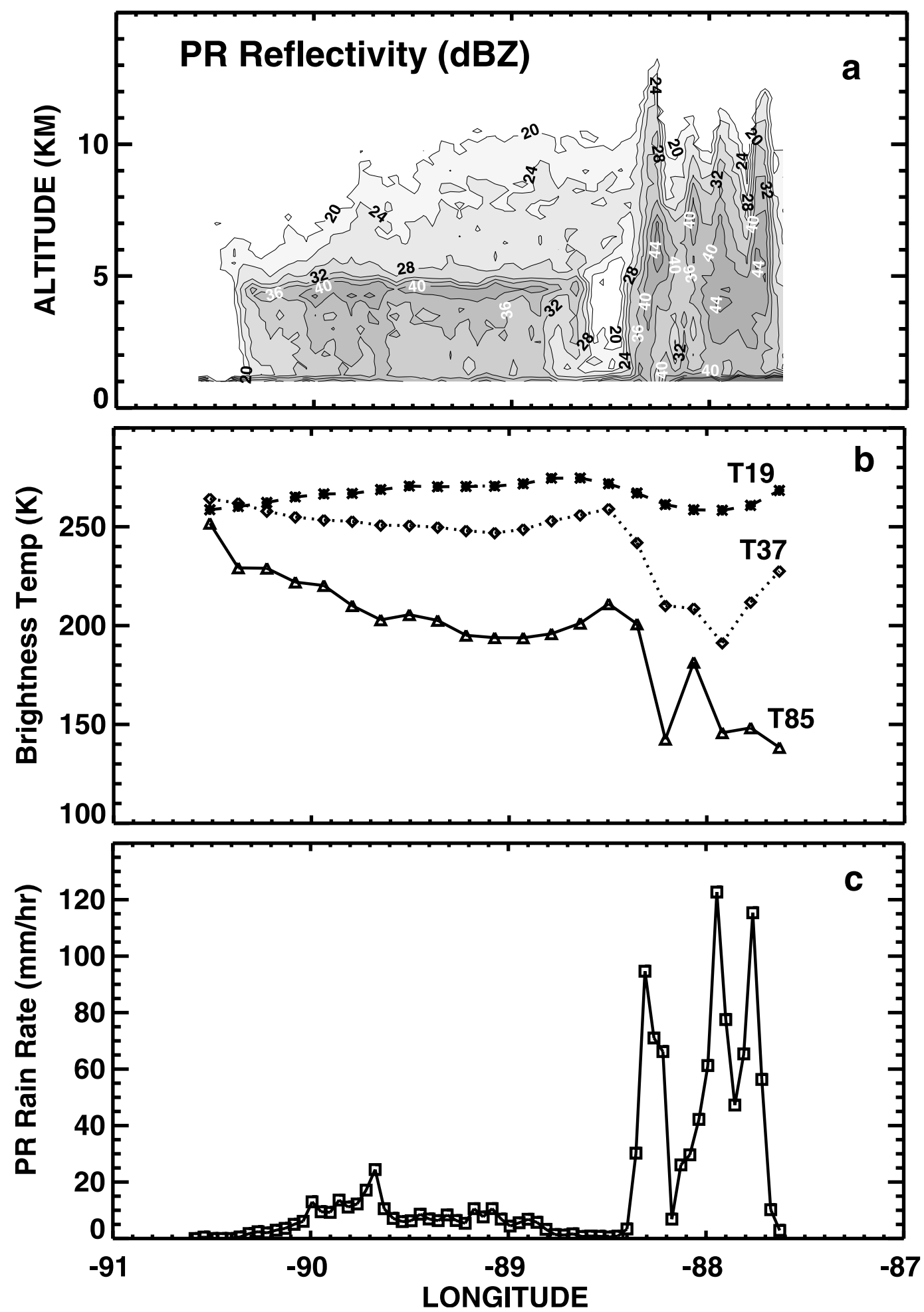

Fig. 2. Vertical cross-sections of a) PR reflectivity, b) TMI T19, T37, and T85, and c) PR nearsurface rain rate for the MCS presented in Figure 1 (see text for details). 

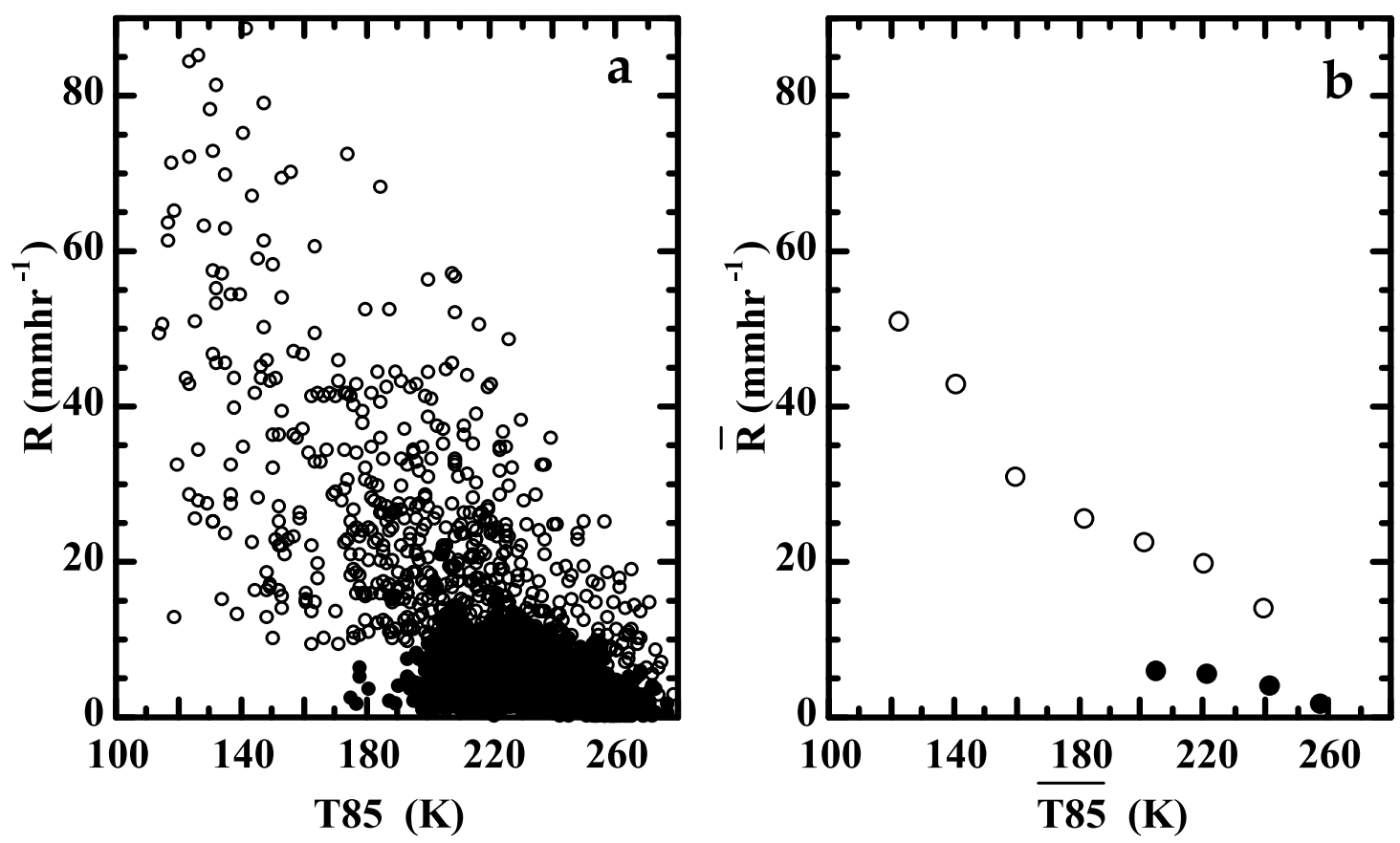

Fig. 3. Observed PR rain rate versus $85 \mathrm{GHz}$ brightness temperature for a) pixels (see text) and b) averages of pixels over $20 \mathrm{~K}$ intervals of T85. The open circles denote observations from convective regions, while the filled circles represent observations from stratiform regions. Data for these plots are taken from TRMM observations of 20 MCS cases over land.

channels. In this study, we have assumed that simple averaging of the TMI 85 and $37 \mathrm{GHz}$ data to the $19 \mathrm{GHz}$ fov also minimizes this spatial mismatch. A similar averaging is also applied to the PR measured reflectivity, $Z$, at several discrete altitudes and to the nearsurface rain rate, $R$. All of these TMI and PR data averaged to the $19 \mathrm{GHz}$ fov are referred to from hereafter as pixel data.

The PR data show that rain rate can be highly variable within a $19 \mathrm{GHz}$ fov. There can be about $30 \mathrm{PR}$ footprints of $4.3 \mathrm{~km} \times 4.3 \mathrm{~km}$ in this fov. These 30 footprints can be sorted according to the PR classification as having stratiform rain or convective rain. In addition, a small number of these PR footprints could be rain free, or have rain that does not fit in the above two categories. Here we have adopted the PR classification of convective and stratiform rain given in the Version 5 of the 2A25 PR data sets (see Iguchi et al. 2000). Also, we assume PR-observed, near-surface rain rate is zero when it is less than $0.1 \mathrm{mmhr}^{-1}$. In this study, a given pixel is categorized as convective when $50 \%$ or more of its area is covered by rain of convective type. This threshold of $50 \%$ is chosen because convective rain area tends to have a small scale. Similarly, a pixel is categorized as stratiform when $80 \%$ or more of its area is covered by stratiform type of rain. For the purpose of generating a set of TMI and PR data associated with such convective and stratiform pixels, we have utilized 20 MCS rain events observed by the TRMM satellite over diverse land regions of the tropics (Africa, Asia, Australia, North America, and South America). Note, to reduce contamination from wet land surfaces, the data where the polarization difference between the vertical and horizontal channels in the $19 \mathrm{GHz}$ is greater than $5 \mathrm{~K}$ are eliminated.

In Fig. 3a, we present a plot of T85 and the corresponding $\mathrm{PR}$ rain rate that have been averaged to TMI $19 \mathrm{GHz}$ fov. This data is gathered from the chosen 20 MCS events. From this plot, we see that the pixels characterized as convective by the PR have relatively high rain rates, and the pixels characterized as strati- 
Table 1. Average of PR and TMI pixel observations (see text) within a given $20 \mathrm{~K}$ interval of T85 for a) convective and b) stratiform rain. The averages are calculated for the following parameters: T85; T37; T19; convective rain fraction $(C)$; stratiform rain fraction $(S)$; PR near-surface rain rate, $R$; and PR reflectivity, $Z$, at 1, 3, 4, 4.5, 6, 7, 9, and $11 \mathrm{~km}$. The number of $20 \mathrm{~km}$ pixel observations in a given T85 interval is also shown in these tables.

a) Convective Rain Pixel Statistics

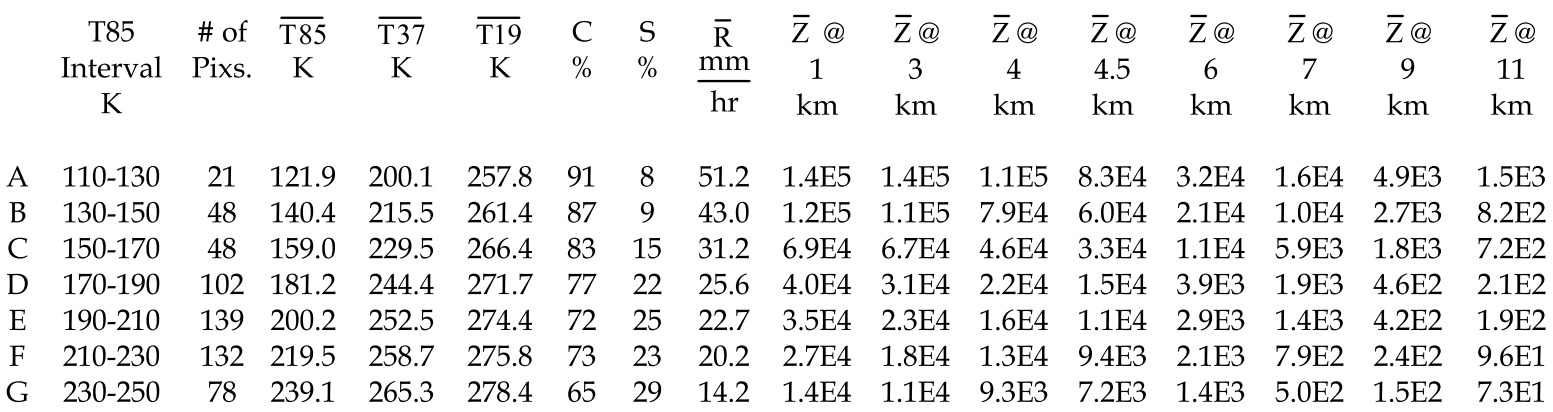

b) Stratiform Rain Pixel Statistics

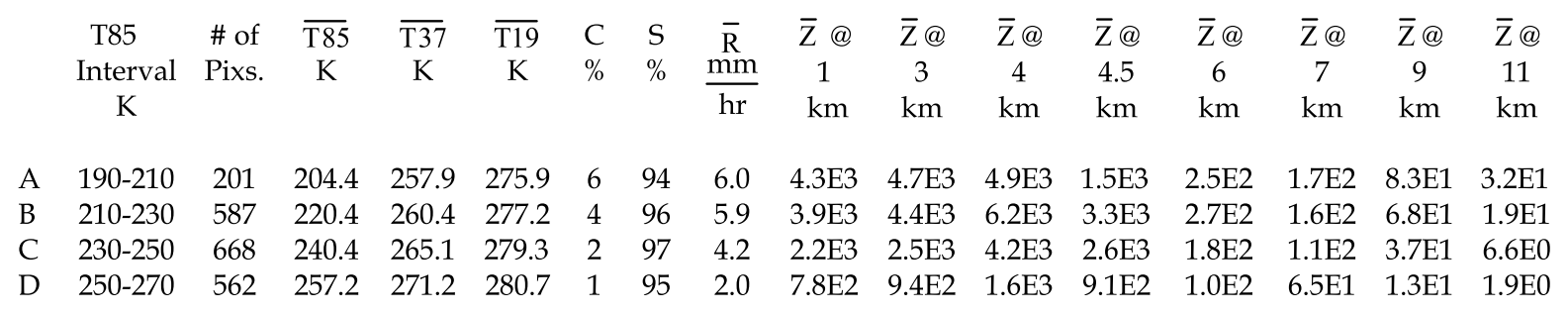

form have generally low rain rates. A closer examination of the PR data shows that most of the convective pixels have rain rates greater than $10 \mathrm{mmhr}^{-1}$, even at warm brightness temperatures as high as $260 \mathrm{~K}$. In the stratiform pixels, more than $97 \%$ have rain rates less than $10 \mathrm{mmhr}^{-1}$. There is a lot of scatter in the data, however, T85 decreases as $R$ increases.

To enhance further statistical strength in the pixel data described above, we have performed additional averaging. First the PR and TMI pixel data are sorted according to the T85 value. Then in each $20 \mathrm{~K}$ interval of T85, averages of the TMI T19, T37, and T85 pixel data are calculated. Similar averages of the near surface rain rate given by $P R$ and the reflectivity, $Z$, at individual altitudes are calculated. This has been done separately for convective and stratiform regions using the data from the chosen 20 MCS events. These averages of pixel data are presented in Tables 1a and $1 \mathrm{~b}$, and are represented with an overbar, i.e., T19, T37, T85, $\bar{Z}$ and $\bar{R}$. Each row in these tables represents a sequential $20 \mathrm{~K}$ interval in T85 and is identified alphabetically. The number of pixels that are averaged in each row is also shown in the tables. It may be pointed out that the standard deviation of all observations of $Z$ at each altitude for a given T85 interval of $20 \mathrm{~K}$ has a magnitude that is comparable to the mean value itself. For each row in Tables 1a and $1 \mathrm{~b}$, the fractional area of convective rain $(C)$ and stratiform rain $(S)$ is indicated as a percentage. Also, since these two rain areas do not add up to $100 \%$, we can infer a small area $(<6 \%)$ with rain that does not fit into the $C$ or $S$ category, or that could be rain free.

Taking the pixel averages given in Tables $1 \mathrm{a}$ and $1 \mathrm{~b}$, we present in Fig. $3 \mathrm{~b}$ the relation-

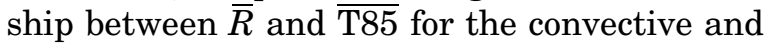
stratiform regions. This figure shows clearly that when one averages the data in the above manner, the variability in the data is reduced, and a clear relationship between $\bar{R}$ and $\overline{\text { T85 }}$ emerges. The convective rain rate increases non-linearly as $\overline{\mathrm{T} 85}$ decreases. This non-linear 

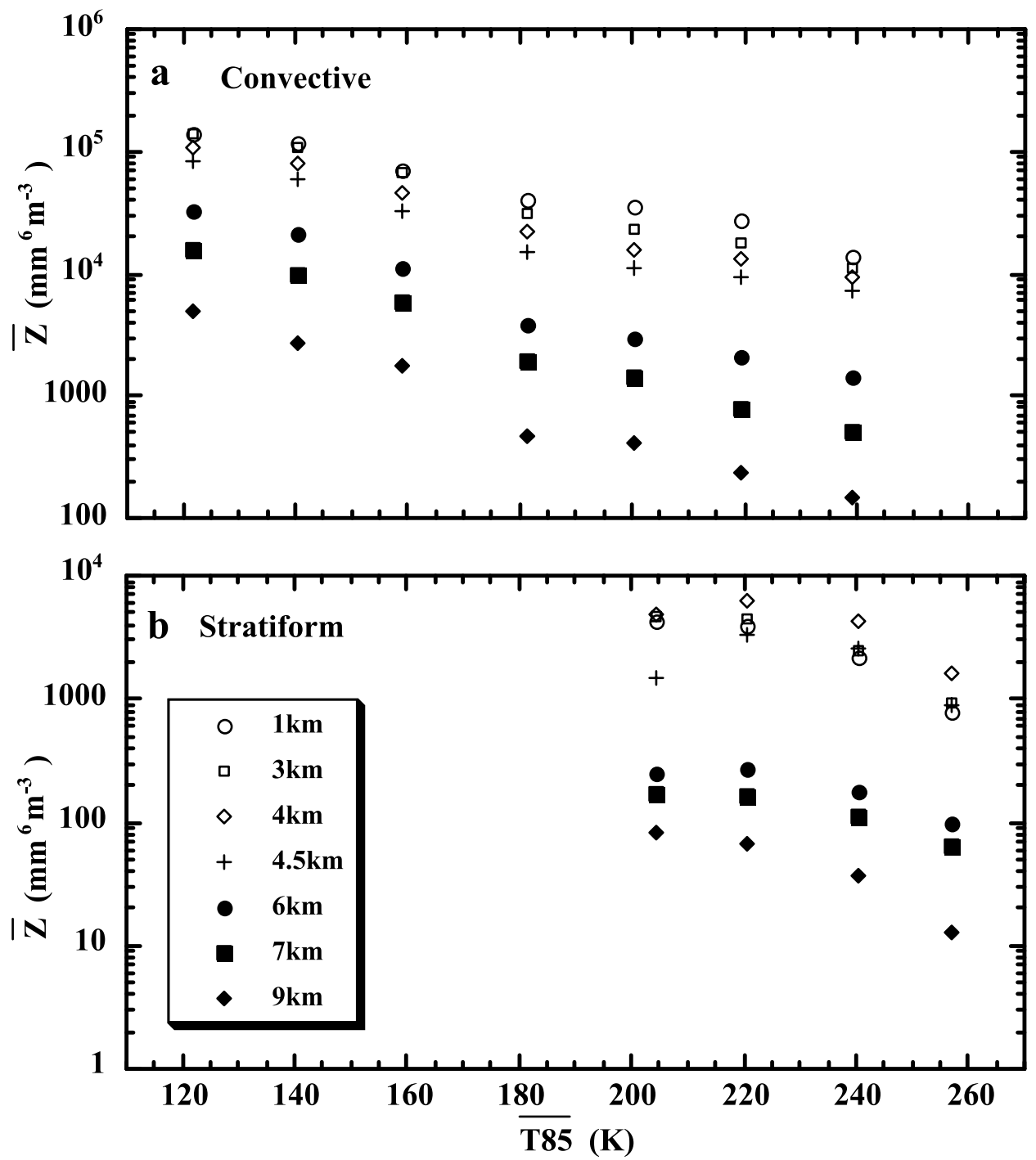

Fig. 4. PR reflectivity, $\bar{Z}$, at different altitudes versus $\overline{\mathrm{T} 85}$ for a) convective and b) stratiform rain.

The symbols denoting the various altitudes are shown as an inset in Panel b.

relationship resembles that derived from radiative transfer theory, where scattering from ice hydrometeors above the freezing level is included (see Wu and Weinman 1984). In the stratiform rain regions, $\overline{\mathrm{T} 85}$ generally does not go below $200 \mathrm{~K}$, and $\bar{R}$ increases slowly as $\overline{\mathrm{T} 85}$ decreases.

In Figs. 4a and 4b, we present plots of $\bar{Z}$ at different altitudes in the atmosphere as a function of T85 using the average pixel data given in Tables 1a and 1b. From Fig. 4a, we find that as T85 increases $\log _{10} \bar{Z}$ decreases almost linearly. In Fig. 4b, where the averages of the pixel data for the stratiform rain are presented, we find again that as $\overline{\text { T85 }}$ increases $\log _{10} \bar{Z}$ decreases almost linearly. Note, in the stratiform rain areas that the magnitude of $\bar{Z}$ in the reflectivity profiles decreases below the melting layer, which is close to $4 \mathrm{~km}$. This decrease is apparently due to evaporation of rain drops below the freezing level.

In convective rain, the PR and TMI observations shown in Fig. 4a demonstrate that there is a relatively strong relationship between T85 and the vertical profiles of $\bar{Z}$. The brightness temperatures $\overline{\mathrm{T} 19}$ and $\overline{\mathrm{T} 37}$ have a weaker rela- 
tionship with the radar reflectivity as shown in Table 1a. From this table, we note that the range in value of $\overline{\mathrm{T} 85}, \overline{\mathrm{T}} 37$ and $\overline{\mathrm{T} 19}$ from Row A to Row $\mathrm{G}$ is about $117 \mathrm{~K}, 65 \mathrm{~K}$, and $21 \mathrm{~K}$, respectively. The corresponding range in $\bar{R}$ is about $37 \mathrm{mmhr}^{-1}$. In addition, the rain rate and Tbs in all three channels vary systematically from Row A to Row G, with no minimum or maximum in between. In stratiform regions, a similar but weaker systematic nature is found.

Based on the averages of the pixels presented in Tables $1 \mathrm{a}$ and $1 \mathrm{~b}$, we note that for a given set of brightness temperatures-i.e., $\overline{\mathrm{T} 19}, \overline{\mathrm{T}} 37$ and T85-one can get widely different rain rates depending on the type of rain that is being observed. An example of this can be found by comparing the data in Row F in Table 1a and Row B in Table 1b. From these rows, we find $\overline{\mathrm{T}} 85$ is close to $220 \mathrm{~K}$, while $\overline{\mathrm{T}} 37$ and $\overline{\mathrm{T} 19}$ are close to $259 \mathrm{~K}$ and $276 \mathrm{~K}$, respectively. However, we find near-surface rain rate, $\bar{R}$, deduced from PR for the convective rain is $20.2 \mathrm{mmhr}^{-1}$, while it is $5.9 \mathrm{mmhr}^{-1}$ for the stratiform rain. Thus, based solely on the brightness temperatures in the 85,37 , and $19 \mathrm{GHz}$ channels, one cannot infer satisfactorily the rain rate unless the type of rain, i.e., convective or stratiform, is known. From this evidence, in order to estimate rain rate from TMI, it is critical to specify the nature of rain from some other information.

From the analysis of the nearly simultaneous TMI and PR observations presented above, we conclude that there is a relationship between them. One such relationship is found for convective rain regions, and another for stratiform rain regions (see Figs. 3a and $3 \mathrm{~b}$ ). In order to vividly show this connection between the TMI and PR data, we have substantially averaged them to minimize the variability introduced by the differences in the scan patterns and viewing geometry of the two instruments, as well as inhomogeneities of the hydrometeors in the atmosphere. In the following section, taking the average properties of the vertical profile of $Z$ given by $\mathrm{PR}$, we are attempting to simulate, using a radiative transfer model, the average brightness temperatures observed by TMI.

\section{Radiative transfer simulations}

In our radiative transfer model we assume local thermodynamic equilibrium and plane- parallel stratification, and it includes absorption and multiple scattering processes. Details pertaining to the formulation of the radiative transfer model, including the vertical profiles of the hydrometeors and their extinction properties, and the numerical calculation procedures are elaborated in a NASA Technical Memorandum by Prabhakara et al. (2002). Here, only the essential features of the radiative transfer model and the key results are presented. The important information that is needed in these radiative transfer calculations is related to the vertical profiles of the hydrometeors for the convective and stratiform rain areas.

\subsection{General considerations}

The basic strategy that we have implemented for both the convective and stratiform models is to simulate the brightness temperatures T85, T37, and T19 for each profile of reflectivity, $\bar{Z}$, given in Tables $1 \mathrm{a}$ and $1 \mathrm{~b}$. Then, these simulated Tbs are compared with the observed $\overline{\mathrm{T} 85}$, $\overline{\mathrm{T} 37}$, and $\overline{\mathrm{T} 19}$ to establish the physical relationship between the TMI and PR measurements. In our models, the radar reflectivity is given by

$$
\bar{Z}=C \int D^{6} N(D) d D,
$$

where $N(D)\left[\mathrm{m}^{-4}\right]$ is the number density of particles in a given size range $d D[\mathrm{~m}]$, and $D$ $[\mathrm{m}]$ is the particle diameter. In the case of snow or graupel, the particle diameter is represented by the equivalent melted diameter (see Ulaby et al. 1981). The constant $C$, which depends on the refractive index, differs between water and ice particles. For water, $C$ has a value of 1.0 , while $C$ is $0.19 / 0.93$ for ice. The particle size distribution (PSD) is described using an exponential function, as given below:

$$
N(D)=N_{0} \exp (-\lambda D) .
$$

In this equation, $N_{0}\left[\mathrm{~m}^{-4}\right]$ and $\lambda\left[\mathrm{m}^{-1}\right]$ are the intercept and slope parameters of the PSD. The parameter $N_{0}$ is given a value based on observations, such as those of Houze et al. (1979) and Sekhon and Srivastava (1970). Then, in order to match $\bar{Z}$ measured by $\mathrm{PR}$, an appropriate value of $\lambda$ is determined with an iterative procedure that utilizes Equations 1 and 2. Since the nature of the hydrometeor profile is different in the convective and stratiform regions, 
the radiative transfer models applicable to these two regions differ.

\subsection{Model and results for convective rain region}

In our convective model, the surface temperature over tropical land is set at $302 \mathrm{~K}$, the temperature lapse rate is $6.0 \mathrm{Kkm}^{-1}$, and for simplicity the surface emissivity is assumed to be 1.0. This gives a freezing level close to 5.0 $\mathrm{km}$. The water vapor distribution in this atmosphere is taken such that the relative humidity is $80 \%$ between the surface and cloud base, and $100 \%$ above. The cloud base is set at $1.5 \mathrm{~km}$, and the cloud liquid water content below the freezing level is assumed to increase linearly from $0.5 \mathrm{gm}^{-3}$ to $1.0 \mathrm{gm}^{-3}$ as surface rain rate increases from $2.0 \mathrm{mmhr}^{-1}$ to $50.0 \mathrm{mmhr}^{-1}$. Cloud liquid water content of such magnitude was reported in hurricanes in a study by Jorgensen (1985).

In the convective region, the PSD at all model levels is inferred using a single value of $N_{0}$. Below the freezing level, all hydrometeors are assumed to be water drops. Above the freezing level, we assume that there is a mixedphase layer. Analyses of differential reflectivity $\left(Z_{D R}\right)$ and linear depolarization ratio (LDR) observations made by polarimetric radars in several other studies (Smith et al. 1999; Bringi et al. 1997; Balakrishnan and Zrnic 1990; and Sax and Keller 1980) lead us to infer a layer of mixed-phase particles, consisting of water and ice, to a substantial height above the freezing level, because of vigorous vertical motions in convective updrafts. The relatively strong reflectivity ( $>30 \mathrm{dBZ}$ ) observed by PR in convective towers above the freezing level (see Fig. 2a) also supports this inference. The detailed nature of these mixed-phase particles-i.e., the fraction and distribution of water and ice in them-is not considered in this study, because of insufficient information. For this reason, in our convective model, we assume for simplicity that there is a mixed-phase layer of finite depth above the freezing level where water drops and frozen graupel exist together in some proportion. The density of water drops and graupel is respectively $1.0 \mathrm{gcm}^{-3}$ and $0.4 \mathrm{gcm}^{-3}$. The fraction of reflectivity due to water drops $\left(f_{\text {wat }}\right)$ and graupel $\left(f_{\text {ice }}\right)$ at each level in the mixedphase layer is given as follows:

$$
\begin{aligned}
f_{\text {ice }} & =\left(\frac{h-h_{F}}{H}\right)^{0.25} \quad \text { where } h_{F}<h<h_{T} \\
f_{\text {wat }} & =1-f_{\text {ice. }}
\end{aligned}
$$

Here $h_{F}$ is the height of the freezing level, and $h$ is the height of any given level in the atmosphere. Now, if we denote the height of the top of the mixed-phase layer as $h_{T}$, then the thickness $H$ of that layer is given by $H=h_{T}-h_{F}$. Equation 3 leads to $100 \%$ liquid phase at the freezing level, which nonlinearly approaches $0 \%$ as $h$ tends to $h_{T}$. Above this mixedphase layer, we assume all hydrometeors are completely frozen graupel with a density of $0.4 \mathrm{gcm}^{-3}$.

Given $N_{0}$ and $H$, we can define the $N(D)$ for the ice and water particles at each level based on the $\bar{Z}$ profile and Eqs. 1, 2 and 3. With this information, we can calculate the Mie volume absorption and scattering coefficients, $k_{a}$ and $k_{s}$, as well as the phase function, $\mathbf{P}$, as a function of height using the procedure developed by Wiscombe (1980). At each level in the mixedphase layer, the total volume absorption coefficient is assumed to be the sum of those of ice, water, and gaseous components. Similarly, the total volume scattering coefficient is given by the sum of those of ice and water. Furthermore, the phase function at each level in the mixedphase layer is assumed to be a combination of the phase functions of ice and water, weighted by their proportions. We may note the properties $k_{a}, k_{s}$ and $\mathbf{P}$ depend on the real and imaginary parts of the refractive index of water and ice. For graupel the values of refractive index are computed as a function of the particle density, which depends on the volume fraction of air and ice (Liao and Meneghini 2000). This information allows us to calculate numerically Tbs at the top of the atmosphere for a particular viewing angle of the radiometer with the help of the radiative transfer equation and boundary conditions. The procedure described above is utilized to simulate the observed $\overline{\mathrm{T} 85}$, $\overline{\mathrm{T} 37}$, and T19 with the $\bar{Z}$ profile given in each row (see Table 1a) and selected combinations of $N_{0}$ and $H$.

In Figs. 5a and 5b, we have plotted T85, simulated with the convective model, as a function of the observed T85 given in each row of Table 1a. In each figure panel, we show three 
curves, which represent T85 simulated using three different values of the depth, $\mathrm{H}$, of the mixed-phase layer above the freezing level (1, 3 , and $5 \mathrm{~km}$ ). Furthermore, in order to show the dependence of these results on $N_{0}$, in Fig. 5 a we utilize a $N_{0}$ value of $2.0 \times 10^{6} \mathrm{~m}^{-4}$, while in Fig. $5 \mathrm{~b}, N_{0}$ is assumed to be $2.0 \times 10^{7} \mathrm{~m}^{-4}$. To appreciate the effectiveness of these simulations, we present in each one of these figure panels a line labeled $\mathbf{A}$, which corresponds to the observed $\overline{\mathrm{T}} 85$.

We find from Figs. 5a and 5b, that as the depth, $H$, of the mixed-phase layer increases, the curves of simulated T85 tend to warmer values, because of reduced ice scattering. Varying $N_{0}$ also has an effect on the simulated T85 values. As the parameter $N_{0}$ decreases, the simulated curves increase in temperature with respect to line $\mathbf{A}$. In view of the several assumptions pertaining to the nature and vertical distribution of hydrometeors introduced in the model, we can only indicate rough values of $N_{0}$ and $H$ that can produce reasonable agreement with observations of T85. On this basis, we contend that a value of $N_{0}$ between $2.0 \times 10^{6} \mathrm{~m}^{-4}$ and $2.0 \times 10^{7} \mathrm{~m}^{-4}$, and a value of about $3 \mathrm{~km}$ for $H$, can simulate $\overline{\mathrm{T} 85}$ in a crude manner.

From the simulations performed for the convective regions, we find that the $85 \mathrm{GHz}$ brightness temperatures given in Table 1a can be simulated crudely. This is because the $85 \mathrm{GHz}$ channel has a fine footprint and strong extinction. As a result, the response in this channel comes primarily from the layers of the atmosphere above the freezing level. In contrast, because of weaker extinction in the longer wavelength channel at $37 \mathrm{GHz}$ significant part of the signal in this channel comes from layers of the atmosphere below the freezing level, and also from the surface. In addition, inhomogeneity of hydrometeors is more pronounced in the broader footprint of this channel. This can introduce appreciable complexity in the data observed in this channel. In the data of the $19 \mathrm{GHz}$ channel, which has even weaker extinction and a broader footprint compared to the $37 \mathrm{GHz}$, these problems are compounded further. For these reasons, radiative transfer simulations in these longer wavelength channels for convective regions (see Prabhakara et al. 2000) are not as good as

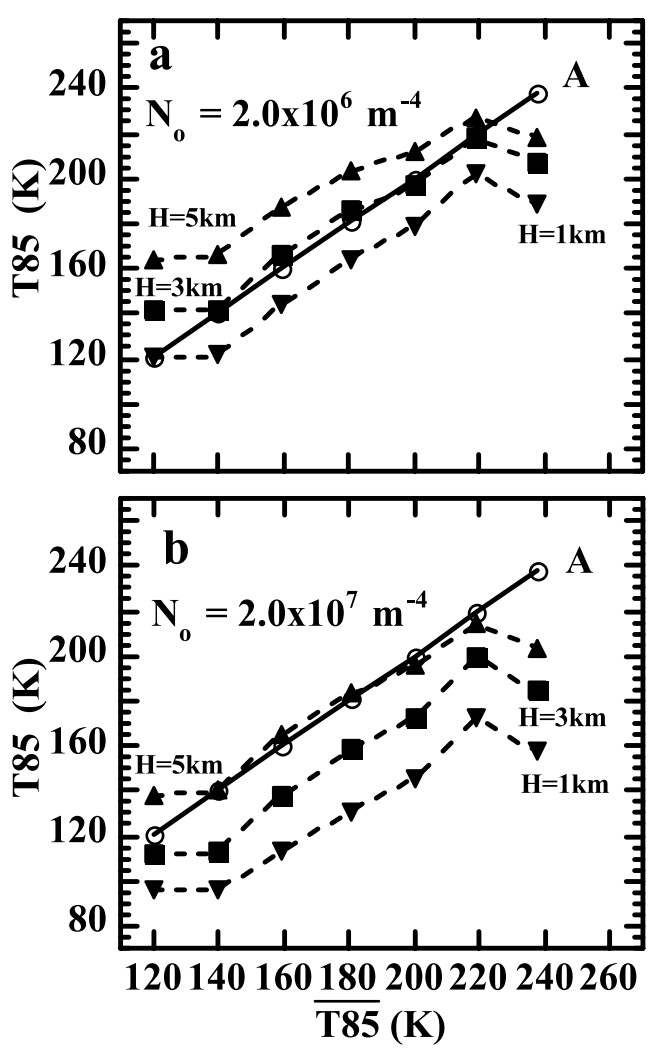

Fig. 5. For the convective regions, simulated T85 versus $\overline{\mathrm{T} 85}$ for the following two values of $N_{0}$ : a) $2 \times 10^{6} \mathrm{~m}^{-4}$ and b) $2 \times 10^{7} \mathrm{~m}^{-4}$. In each figure, the depth, $H$, of the mixed-phase layer above the freezing level is indicated for each curve. The solid line $\mathbf{A}$ in these figures representing $\overline{\mathrm{T} 85}$ observations is included for comparison.

those for the $85 \mathrm{GHz}$ channel. However, when the difference, (T19-T37), of the simulations of T19 and T37 is considered, these undesirable effects are substantially reduced. This is demonstrated in Figs. 6a and 6b.

\subsection{Model and results for stratiform rain region}

In the stratiform model, the surface emissivity and vertical profile of temperature are the same as that in the convective model. The water vapor distribution in this atmosphere is taken such that the relative humidity is $80 \%$ between the surface and the freezing level, and $100 \%$ above. At altitudes above the freezing level $(\sim 5.0 \mathrm{~km})$, only ice aggregates (snow 


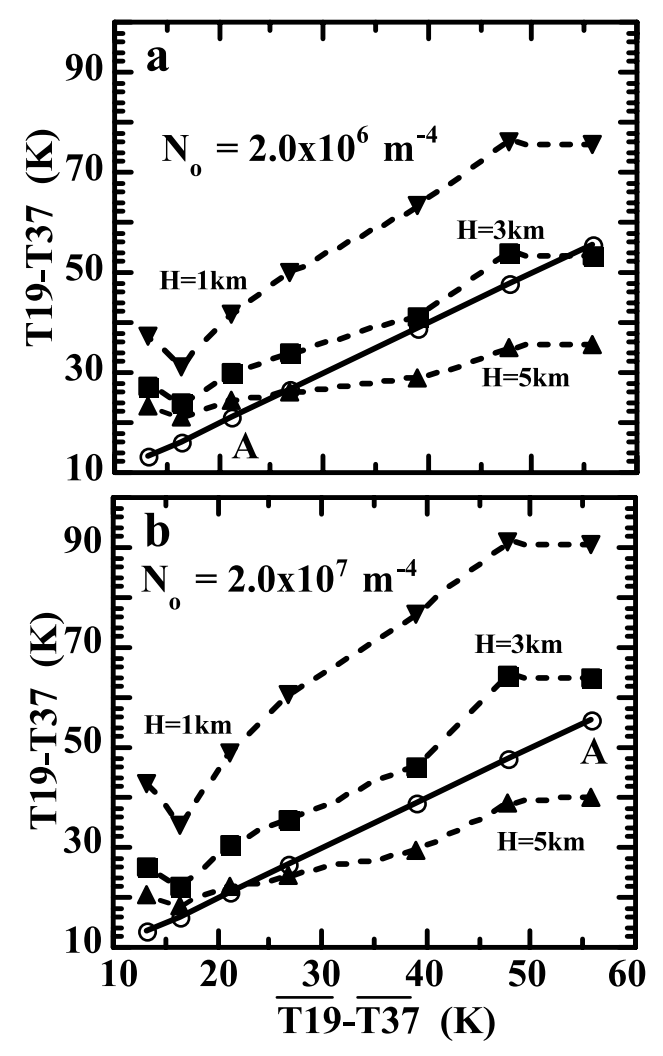

Fig. 6. For the convective regions, simulated T19-T37 versus T19-T37 for the following two values of $N_{0}$ : a) $2 \times 10^{6}$ $\mathrm{m}^{-4}$ and b) $2 \times 10^{7} \mathrm{~m}^{-4}$. In each figure, the depth, $H$, of the mixed-phase layer above the freezing level is indicated for each curve. The solid line $\mathbf{A}$ in these figures representing $\overline{\mathrm{T} 19}-\overline{\mathrm{T}} 37$ observations is included for comparison.

flakes) are assumed to exist. The density of ice aggregates, $\rho_{\text {ice }}$, is given as follows:

$$
\rho_{\text {ice }}=\frac{2.0}{D_{\mathrm{sn}}^{2}},
$$

where $D_{\text {sn }}[\mathrm{mm}]$ is the diameter of a spherical particle whose volume is equal to the volume of the ice aggregate. Equation 4 is based on a similar expression developed by Magono and Nakamura (1965) (see also Schols et al. 1999). In Eq. 4, the limiting value of $\rho_{\text {ice }}$ is set equal to $0.7 \mathrm{gcm}^{-3}$, as was done by Schols et al.. For the stratiform simulations, we have developed an empirical model for the intercept parameter, $N_{0}$, of the PSD above the freezing level. This model is based on the observational studies of
Houze et al. (1979) and Sekhon and Srivastava (1970). Houze et al. showed that $N_{0}$ increases as the atmospheric temperature decreases, while Sekhon and Srivastava revealed that in the stratiform region $N_{0}$ decreases as water content in the atmosphere increases. Based on this information, we have developed an empirical equation to give $N_{0}$ as a function of height above the freezing level in the atmosphere as follows:

$$
N_{0}(R, h)=N^{*} \exp \left(R^{-0.94}+\left(h-h_{F}\right)\right),
$$

where $h-h_{F}$ is the height, in kilometers, above the freezing level and $R$ is rain rate. In Equation 5 , the argument of the exponential function is forced not to exceed a value of 3.0. Also, the value of $N_{0}$ is not allowed to exceed $3 \times 10^{8} \mathrm{~m}^{-4}$. This maximum value of $N_{0}$ is consistent with the observations of Houze et al. (1979). Below the freezing level, $N_{0}$ is given as

$$
N_{0}(R)=N^{*} \exp \left(R^{-0.94}\right) .
$$

The value of the parameter $N^{*}$ in Eqs. 5 and 6 is varied over a range between $10^{5} \mathrm{~m}^{-4}$ and $10^{7} \mathrm{~m}^{-4}$ to evaluate the impact of this parameter on the upwelling brightness temperature.

In our stratiform model, we have a layer of relatively-large, slowly-falling, low-density, melting snow flakes below the freezing level. The depth of the melting layer is taken to be $500 \mathrm{~m}$. To estimate the refractive index of these melting snow flakes, we have adopted the Maxwell-Garnet mixing model of ellipsoidal ice inclusions randomly distributed in a homogeneous water matrix (Meneghini and Liao 1996 and 2000). In this combination, the water contribution dominates for all meltwater fractions (Bauer et al. 2000). In our stratiform model, it is assumed that these snow flakes melt completely and form raindrops at the base of the melting layer.

The hydrometeor profiles described above for the stratiform rain model are constructed to be consistent with the stratiform profiles of $\bar{Z}$ given in Table 1b. As in the convective model, we compute the Mie volume extinction and scattering coefficients, as well as the phase function, as a function of height for these hydrometeor profiles. This information is used to calculate Tbs at the top of the atmosphere for a particular viewing angle of the radiometer with the help of the radiative transfer equation and 


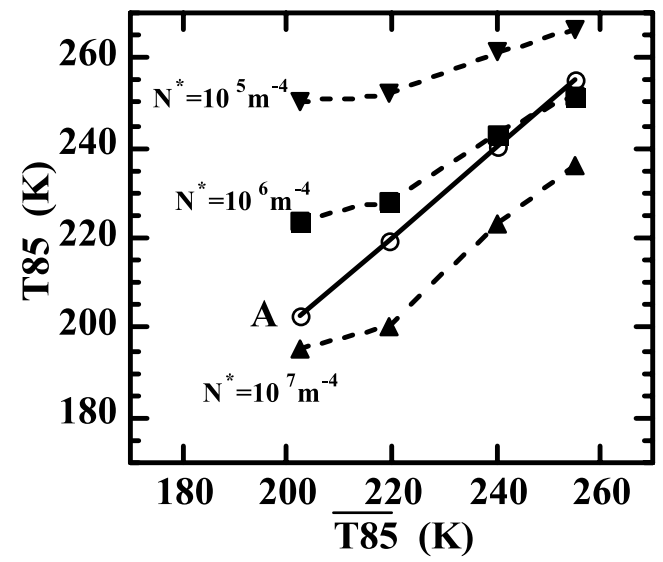

Fig. 7. For the stratiform regions, dashed lines indicate simulated T85 versus observed $\overline{\mathrm{T} 85}$ for the following three values of $N^{*}: 10^{5}, 10^{6}$, and $10^{7} \mathrm{~m}^{-4}$. For comparison, the solid line $\mathbf{A}$ representing the observations $\overline{\mathrm{T} 85}$ are presented in the figure.

boundary conditions. From the description of the hydrometeor profile, we note that $N^{*}$ is the key parameter for the stratiform region.

In Fig. 7, we are presenting the results obtained from the simulations pertaining to the stratiform observations presented in Table $1 \mathrm{~b}$. Three curves (dashed lines) shown in this figure correspond to simulations based on three different values of $N^{*}\left(10^{5}, 10^{6}\right.$, and $\left.10^{7} \mathrm{~m}^{-4}\right)$. In addition, a line labeled $\mathbf{A}$ represents observed brightness temperatures. The $85 \mathrm{GHz}$ simulations presented in Fig. 7 show that when $N^{*}$ is between $10^{6}$ and $10^{7} \mathrm{~m}^{-4}$, a crude agreement between the observed $\overline{\mathrm{T} 85}$ and simulated T85 can be obtained. However, in view of the various limitations, our model fails to simulate the weak range in $\overline{\mathrm{T} 19}-\overline{\mathrm{T}} 37$ in the stratiform region. The difference, ( $\overline{\mathrm{T} 19}-\overline{\mathrm{T}} 37)$, in the stratiform rain regions has a relatively small range between $9 \mathrm{~K}$ and $18 \mathrm{~K}$ (see Table 1b). By contrast, we may point out that the range of $\overline{\mathrm{T} 19}$ T37 for the convective rain is about $13 \mathrm{~K}$ to $58 \mathrm{~K}$, which we are able to crudely simulate (see Table 1a).

From the radiative transfer simulations presented above using convective and stratiform models, we conclude it is possible to crudely explain the interrelationship between $\overline{\mathrm{T} 85}$ observed by the radiometer and $\bar{Z}$ profiles mea- sured by the radar. However, only the difference $\overline{\mathrm{T} 19}-\overline{\mathrm{T}} 37$ can be explained for the convective region. Mainly, in these simulations, a given reflectivity profile is linked to the observed Tbs through the profile of the water content and the prescribed microphysics. The microphysical properties are based on observational studies made by prior investigators using aircraft and radars measurements.

From Table 1a, one finds for convective regions that the average PR reflectivity at all levels in the atmosphere increases systematically as the average near-surface rain rate increases. This systematic relationship explains the connection between T85 and T19-T37 with the average near-surface radar rain rate for these regions. For stratiform regions, from Table $1 b$, one finds that the average PR reflectivity at all levels in the atmosphere does not increase systematically as the average near-surface rain rate increases. This is because of significant evaporation of hydrometors in the stratiform rain regions (Houze 1997). This introduces some difficulty in relating $\overline{\mathrm{T} 85}$ and (T19-T37) with the weak average nearsurface radar rain rate in the stratiform regions.

\section{Conclusions}

Hydrometeor distributions associated with rain are highly variable in space and time. Understanding the impact of hydrometeor profiles on radiative transfer with the help of observations and models is needed in developing rain retrieval methods from microwave radiometer data. For this purpose, in this study we have examined the information given by the PR and TMI onboard the TRMM satellite for relatively pure convective and stratiform rain regions. A simple evaluation of the PR and TMI data shows poor correspondence of the information given by them. This is because of differences in the scan patterns and viewing geometry of the two instruments, as well as inhomogeneities of the hydrometeors in the atmosphere. For this reason, we average the PR and TMI data significantly. The average properties of the PR and TMI data presented in Tables $1 \mathrm{a}$ and $1 \mathrm{~b}$, based on 20 MCS cases over tropical land, are very helpful to compare the observations from these two instruments. These tables, and Figs. $4 \mathrm{a}$ and $4 \mathrm{~b}$, show that on average there is a re- 
lationship between the PR reflectivity profiles and TMI measured brightness temperatures in 85, 37, and $19 \mathrm{GHz}$. Also, TMI and PR observations in the tropics show that there are two distinctly different relationships between T85 and $P R$ rain rate in convective and stratiform rain regions. As shown in Fig. 3b, the slope of $\bar{R}$ with respect to $\overline{\text { T85 }}$ is large for convective regions, and small for stratiform regions. Furthermore, from Tables $1 \mathrm{a}$ and $1 \mathrm{~b}$, one finds that the radiometer data in 19,37 , and $85 \mathrm{GHz}$ channels on the average have a poor ability to discriminate convective and stratiform rain areas. For this reason, a method to discriminate crudely these rain areas was developed in a study by Prabhakara et al. (2000). That method is based on identification of local minima in the spatial distribution of T85.

In order to explain the relationship between TMI measured brightness temperatures and PR reflectivity profiles (see Figs. $4 a$ and $4 b$ ), we have performed radiative transfer calculations as discussed in Section 3. In these calculations, we are able to crudely simulate the $85 \mathrm{GHz}$ brightness temperatures in convective and stratiform regions. The simulated values of T19-T37 for the convective rain region can give similar agreement as in the case of the $85 \mathrm{GHz}$. This suggests that T19-T37 may be used as additional information to T85 to improve rain retrieval from TMI on land.

Liu and Curry (1998, 1999, and 2000) show from aircraft and satellite passive microwave radiometer data that channels near $150 \mathrm{GHz}$, which have much stronger extinction than the $85 \mathrm{GHz}$, can give useful information about ice water content in the upper layers of the troposphere. Because of its higher frequency, for the same size of antenna, a channel near $150 \mathrm{GHz}$ can yield a spatial resolution that is about two times better than the $85 \mathrm{GHz}$. We find that this high extinction and spatial resolution will be extremely valuable in isolating brightness temperature minima corresponding to convective cells in vigorously developing and decaying thunderstorms. Also, $150 \mathrm{GHz}$ radiation is more opaque to water vapor than that at 85 GHz. This makes a $150 \mathrm{GHz}$ channel useful in the middle and high latitudes, where surface contamination becomes a problem at $85 \mathrm{GHz}$. From these considerations, we contend that addition of a $150 \mathrm{GHz}$ channel to microwave radiometers with conical-scanning geometry and with spatially continuous observations will be valuable for the remote sensing of rain.

Understanding the relationship between the measurements made by microwave radiometers and convective and stratiform rain is necessary for the future Global Precipitation Measurement (GPM) mission (E.A. Smith, personal communication). In GPM a constellation of satellites with microwave radiometers are expected to be flown without an accompanying radar. This will also be the case with microwave radiometers onboard the Earth Observing System's Aqua satellite and the Japanese satellite ADEOS-II. Thus, the present study has useful applications for future satellite missions.

\section{Acknowledgments}

We thank the anonymous reviewers for their valuable comments to improve this paper. During this research, Dr. Yoo was supported by the Climate Environment System Research Center sponsored by the SRC program of the Korea Science Engineering Foundation. Also, the TRMM data for this research was supplied by the Distributed Active Archive Center (DAAC) of NASA Goddard Space Flight Center.

\section{References}

Balakrishnan, N. and D.S. Zrnic, 1990: Estimation of rain and hail rates in mixed-phase precipitation. J. Atmos. Sci., 47, 565-583.

Bauer, P., A. Khain, A. Pokrovsky, R. Meneghini, C. Kummerow, F. Marzano, and J.P.V. Poiares Baptista, 2000: Combined cloud-microwave radiative transfer modeling of stratiform rainfall. J. Atmos. Sci., 57, 1082-1104.

Bennartz, R. and G.W. Petty, 2001: The sensitivity of microwave remote sensing observations of precipitation to ice particle size distributions. J. Appl. Meteor., 40, 345-364.

Bringi, V.N., K. Knupp, A. Detwiler, L. Lui, I.J. Caylor, and R.A. Black, 1997: Evolution of a Florida thunderstorm during the Convective and Precipitation/Electrification Experiment: The case of 9 August 1991. Mon. Wea. Rev., 125, 2131-2160.

Haddad, Z.S., E.A. Smith, C.D. Kummerow, T. Iguchi, M.R. Farrar, S.L. Durden, M. Alves, W.S. Olson, 1997: The TRMM 'Day-1' Radar/ Radiometer Combined Rain-Profiling Algorithm. J. Meteor. Soc. Japan, 75, 799-809. 
Hong, Y., J.L. Haferman, W.S. Olson, and C.D. Kummerow, 2000: Microwave brightness temperatures from tilted convective systems. $J$. Appl. Meteor., 39, 983-998.

Houze Jr., R.A., P.V. Hobbs, P.H. Herzegh, and D.B. Parsons, 1979: Size distributions of precipitation particles in frontal clouds. J. Atmos. Sci., 36, 156-162.

1993: Cloud Dynamics. Academic Press, Inc., San Diego, 571 pp.

- 1997 : Stratiform precipitation in regions of convection: A meteorological paradox? Bull. Amer. Meteor. Soc., 78, 2179-2196.

Iguchi, T., T. Kozu, R. Meneghini, J. Awaka, and K. Okamoto, 2000: Rain-profiling algorithm for the TRMM Precipitation Radar. J. Appl. Meteor., 39, 2038-2052.

Jorgensen, D.P., E.J. Zipser, and M.A. LeMone, 1985: Vertical motions in intense hurricanes. J. Atmos. Sci., 42, 839-856.

Liao, L. and R. Meneghini, 2000: Investigation of the dielectric contant of inhomogeneous air-ice and snow-water spheres. IGARSS.

Liu, G. and J.A. Curry, 1998: Remote sensing of ice water characteristics in tropical clouds using aircraft microwave measurements. J. Appl. Meteor., 37, 337-355. and - 1999: Tropical ice water amount and its relations to other atmospheric hydrological parameters as inferred from satellite data. J. Appl. Meteor., 38, 1182-1194. and - 2000: Determination of ice water path and mass median particle size using multi-channel microwave measurements. $J$. Appl. Meteor., 39, 1318-1329.

Meneghini, R. and L. Liao, 1996: Comparisons of cross sections for melting hydrometeors as derived from dielectric mixing formulas and a numerical method. J. Appl. Meteor., 35, 16581670.

$\longrightarrow$ and $\longrightarrow, 2000$ : Effective dielectric constants of mixed-phase hydrometeors. J. Atm. Oceanic Tech., 17, 628-640.

Magono, C., and T. Nakamura, 1965: Aerodynamic studies of falling snowflakes. J. Meteor. Soc. Japan, 43, 139-147.

Prabhakara, C., R. Iacovazzi, Jr., J.A. Weinman, and G. Dalu, 2000: A TRMM microwave radiometer rain rate estimation method with convective and stratiform discrimination. J. Meteor. Soc. Japan, 78, 241-258.

- - , and J.-M. Yoo, 2002: Simulation of TRMM Microwave Imager brightness temperature using Precipitation Radar reflectivity for convective and stratiform rain areas over land. NASA Tech. Memo. \#2002-210000, 56 pp.

Sax, R.I. and V.W. Keller, 1980: Water-ice and water-updraft relationships near $-10^{\circ} \mathrm{C}$ with populations of Florida cumuli. J. Appl. Meteor., 19, 505-514.

Schols, J.L., J.A. Weinman, G.D. Alexander, R.E. Stewart, L.J. Angus, and A.C.L. Lee, 1999: Microwave properties of frozen precipitation around a North Atlantic cyclone. J. Appl. Meteor., 38, 29-43.

Sekhon, R.S. and R.C. Srivastava, 1970: Snow size spectra and radar reflectivity. J. Atmos. Sci., 27, 299-307.

Smith, P.L., D.J. Musil, A.G. Detwiler, and R. Ramachandran, 1999: Observations of mixed-phase precipitation within a CaPE thunderstorm. $J$. Appl. Meteor., 38, 145-155.

Ulaby, F.T., R.K. Moore, and A.K. Fung, 1981: $M i$ crowave Remote Sensing: Active and PassiveVolume I: Microwave Remote Sensing Fundamentals and Radiometry. Artech House, Norwood, MA, 456 pp.

Viltard, N., C. Kummerow, W.S. Olson, and Y. Hong, 2000: Combined use of the radar and radiometer of TRMM to estimate the influence of drop size distribution on rain retrievals. $J$. Appl. Meteor., 39, 2103-2114.

Wiscombe, W., 1980: Improved Mie scattering algorithms. Appl. Opt., 19, 1505-1509.

$\mathrm{Wu}, \mathrm{R}$. and J.A. Weinman, 1984: Microwave radiances from precipitating clouds containing aspherical ice, combined phase, and liquid hydrometeors. J. Geophy. Res., 89, 7170-7178. 